/05/ 


\section{EXELEARNING: RECURSO DIGITAL DE UNA ESTRATEGIA DIDÁCTICA DE ENSEÑANZA-APRENDIZAJE DE MATEMÁTICA}

\section{EXELEARNING: DIGITAL RESOURCE OF A DIDACTIC TEACHING STRATEGY MATH LEARNING}

Verónica Yánez Ortiz

Docente Titular. Responsable de Investigación Escuela de Agroindustrias. Pontificia Universidad

Católica del Ecuador Sede Esmeraldas. Esmeraldas, (Ecuador)

E-mail: veronica.yanez@pucese.edu.ec ORCID: https://orcid.org/0000-0001-7732-8168

Manuel Nevárez Toledo

Docente Titular. Responsable del Laboratorio de Investigación Tecnologías e Innovación. Escuela Ingeniería de Sistemas y Computación. Pontificia Universidad Católica del Ecuador Sede Esmeraldas. Esmeraldas, (Ecuador) E-mail: manuel.nevarez@pucese.edu.ec ORCID: https://orcid.org/0000-0001-5628-3351

Recepción: 22/11/2018. Aceptación: 15/12/2018. Publicación: 28/12/2018

Citación sugerida:

Yánez Ortiz, V. y Nevárez Toledo, M. (2018). Exelearning: recurso digital de una estrategia didáctica de enseñanza-aprendizaje de matemática 3C TIC. Cuadernos de desarrollo aplicados a las TIC, 7(4), pp.98-121. doi:http://dx.doi.org/10.17993/3ctic.2018.62.98-121 


\section{RESUMEN}

La didáctica de la Matemática, en su constante búsqueda por mejorar los procesos de enseñanzaaprendizaje, ha vinculado herramientas tecnológicas educativas, desde los recursos de apoyo a las estrategias de enseñanza para procurar un aprendizaje efectivo de los estudiantes. Con este proyecto se propone determinar si la utilización del software libre educativo eXe-Learning contribuye a mejorar el desempeño académico de los estudiantes de Matemática de primer nivel de la Escuela de Sistemas de la Pontificia Universidad Católica del Ecuador, Sede Esmeraldas. Con un enfoque constructivista se delineó la estrategia didáctica aplicada a una población de 26 estudiantes, divididos aleatoriamente en dos grupos. La investigación es de tipo experimental con diseño post-test con grupo control, la aleatorización garantizó la comparabilidad de los grupos; se aplicó el razonamiento hipotético-deductivo con una metodología cuantitativa para el manejo de los datos generados por las evaluaciones. Los resultados obtenidos, aplicando la prueba de $\mathrm{T}$ de Student, demostraron que la media $(0,8808)$ de las calificaciones de la prueba aplicada a los estudiantes del grupo experimental es mayor que la media $(0,4962)$ de las calificaciones aplicada a los estudiantes del grupo de control. Esta diferencia significativa no es producto del azar e implica que una estrategia didáctica de enseñanza apoyada con el eXe-Learning contribuye a mejorar el desempeño académico de los estudiantes.

\section{ABSTRACT}

The didactic of Mathematics, in its constant search to improve teaching-learning processes, has linked educational technological tools, as support resources or teaching strategies to ensure effective learning of students. With this project it was proposed to determine if the use of free educational software eXe-Learning contributes to improve the academic performance of the students of Mathematics of the first level of the School of Systems of the Pontifical Catholic University of Ecuador, Esmeraldas Campus. With a constructivist approach, the didactic strategy was outlined which was applied, as a pilot test, to a population of 26 students divided randomly into two groups. The investigation was of experimental type with post-test design with control group, randomization guaranteed the comparability of the groups; the hypothetic-deductive reasoning was applied with a quantitative methodology for the management of the data generated by the evaluations. The results obtained, applying the Student's T test, showed that the mean (0.8808) of the test scores applied to the students of the experimental group is higher than the average (0.4962) of the scores applied to the students. of the control group; This significant difference is not a product of chance and implies that a didactic teaching strategy supported by eXe-Learning contributes to improving the academic performance of students. 


\section{PALABRAS CLAVE}

eXe-Learning, Recurso didáctico digital, Estrategia de enseñanza-aprendizaje, Matemática, Software libre.

\section{KEY WORDS}

eXe-Learning, Digital didactic resource, Teaching-learning strategy, Mathematics, Free software. 


\section{INTRODUCCIÓN}

La incorporación de las TIC en los procesos educativos, en la actualidad, es indiscutible y son varias las herramientas tecnológicas desarrolladas con el fin de apoyarlos, sin embargo, ninguna herramienta tecnológica, por sí sola, puede aportar a la educación sin estar ligada a una estrategia didáctica apropiada. Para investigadores como Oyola y Gómez (2012), Ferro y Martínez (2009), Castillo (2008), Mora (2003), entre otros, el proceso de enseñanza aprendizaje se ve fortalecido con el empleo de estrategias didácticas que garantizan el uso apropiado de las tecnologías como recursos educativos.

Dentro de la dinámica pedagógica, el docente, desde una postura constructivista, debe promover procesos educativos que permitan la participación de los estudiantes a través de actividades previamente planificadas y diseñadas para propiciar su desarrollo intelectual (Castro, Peley y Morillo, 2006). En este sentido, es primordial que el docente considere las TIC como un recurso de apoyo que debe insertar al delinear su estrategia didáctica.

Para Ocampo (2003), las estrategias didácticas son procesos o secuencias de actividades que sirven de base a la realización de tareas intelectuales y que se eligen con el propósito de facilitar la construcción, permanencia y transferencia de conocimientos (p. 9). Por otro lado, Carrasco (2004), define una estrategia didáctica de enseñanza como el conjunto de instrucciones que el docente diseña y aplica para beneficiar los procesos de enseñanza-aprendizaje

Bajo este contexto, según Díaz y Hernández (2012), las estrategias didácticas de enseñanza pueden ser: pre-instruccionales, co-instruccionales y post-instruccionales; las que están respectivamente ligadas a diferentes momentos de una clase: inicio, en el que se plantea lo que se va a aprender y cómo se lo hará; desarrollo, en el que se exponen los contenidos y se aplican técnicas motivacionales para mantener el interés de los estudiantes; cierre, en el que se comprueba si los objetivos propuestos al inicio de la clase se han alcanzado.

En el campo de las matemáticas, la enseñanza-aprendizaje debe estar en concordancia con el avance tecnológico, para lo cual, debe estar ligada a una metodología que favorezca el desarrollo cognitivo de los estudiantes, que estimule su pensamiento crítico y su autorregulación, que tienda el puente entre la educación tradicional y aquella que emplea la tecnología a su favor (García, Escalera y Navarro, 2011). 
En el entorno educativo, varios son los recursos tecnológicos que se utilizan, entre ellos: software educativo, simuladores, herramientas multimedia y de presentación, correo electrónico, blogs, etc., algunos de ellos creados específicamente como recursos didácticos y otros que se han adaptado. En concordancia a lo anteriormente expuesto, Herrera, Novelo, Díaz y Hernández (2016), opinan que dentro de las TIC, los software educativos especializados también son herramientas que se pueden tomar como estrategias para la enseñanza-aprendizaje de las matemáticas (p. 8). De igual manera, Morales y Peña (2013) argumentan que el empleo de herramientas tecnológicas educativas para la enseñanza de matemáticas puede ser fructífero si están acompañadas de estrategias apropiadas. En razón de lo mencionado, el uso de cualquiera de estos recursos no es una garantía de que el proceso educativo se lleve adecuadamente y que su efectividad se vea reflejada en los logros alcanzados por los estudiantes; de ahí que es necesario que el docente planifique su clase y establezca la herramienta tecnológica que, de acuerdo con sus características, se adapte mejor a su estrategia didáctica.

Para este proyecto se considera como unidad de estudio el capítulo de lógica matemática, abordando los siguientes temas: proposiciones, conectores lógicos, lectura y escritura lógicas contemplada en el syllabus de Matemática de primer nivel de la Escuela de Sistemas de la PUCE Esmeraldas. Con esta referencia se diseña la estrategia didáctica con un enfoque constructivista, considerando los tres momentos de una clase: inicio, desarrollo y cierre; y contemplando una evaluación tanto formativa como sumativa. En base a los contenidos y actividades a realizar, se considera pertinente emplear como recurso digital de apoyo un software educativo, que permita la creación y manejo de contenidos, que además resulte ser amigable para el usuario; de acceso libre y gratuito. En función de estos requerimientos y tras un análisis previo, se considera que la herramienta tecnológica idónea para apoyar la estrategia es el software educativo eXe-Learning.

Una vez estructurada la estrategia didáctica apoyada con el recurso digital eXe-Learning es importante determinar si su aplicación produce algún efecto en el proceso de enseñanza-aprendizaje de los estudiantes, para ello se plantea la siguiente hipótesis: La media de las calificaciones del test aplicado a los estudiantes, que recibieron las clases con la estrategia apoyada con eXe-Learning es mayor que la media de las calificaciones de aquellos que recibieron las clases sin la estrategia apoyada con eXe-Learning. A continuación, se detalla la metodología empleada, el diseño de la estrategia apoyada con el eXe-Learning, los resultados obtenidos al aplicarla y finalmente se exponen las conclusiones generadas por esta investigación. 


\section{METODOLOGÍA}

La investigación es de tipo experimental, desarrollada en dos etapas: la primera relacionada con la utilización del eXe-Learning como recurso digital para el diseño de una estrategia didáctica de enseñanza, bajo el paradigma constructivista. Aprovechando las características del software, se estructuró la estrategia considerando un árbol de contenidos, plasmando la respectiva información en un sitio web, respetando un orden secuencial a favor de los procesos de enseñanza-aprendizaje; la segunda etapa, concebida para establecer las relaciones de causa-efecto entre las variables generadas, una vez diseñada la estrategia apoyada con un recurso digital como el eXe-Learning, determinó cómo su aplicación influye en el rendimiento académico de un grupo de estudiantes de Matemática de primer nivel universitario, a modo de prueba piloto.

Para la segunda etapa, la metodología experimental se respaldó en un razonamiento hipotético deductivo en base al efecto de la estrategia sobre el rendimiento académico, mediante un experimento controlado, sobre la aplicación de la estrategia a dos grupos de estudiantes elegidos aleatoriamente, fue probada a través de una evaluación tipo cuestionario. Para la comprobación se empleó la metodología cuantitativa mediante técnicas estadísticas inferenciales paramétricas desarrolladas con el apoyo del software SPSS, aplicación útil para el procesamiento y análisis de datos estadísticos.

\subsection{DISEÑO DE LA PRIMERA ETAPA}

Para el desarrollo de la estrategia didáctica se consideró que el estudiante con la información presentada tendría la oportunidad de construir su propio conocimiento, respaldado por su condición de nativo digital (González, 2016), bajo estas circunstancias, se empleó como recurso digital al software educativo eXe-Learning, después de un análisis de las diferentes herramientas tecnológicas que se emplean en la educación y que están disponibles en el entorno de la PUCE Esmeraldas. Como se puede observar en la Tabla 1 el eXe-Learning al ser comparado con Moodle y Microsoft Power Point resulta tener más ventajas.

$\mathrm{Al}$ ser el eXe-Learning una herramienta de autor de código abierto para generar contenidos digitales en formatos diversos: HTML, SCORM, EPUB... se ajustó y contribuyó al diseño de la estrategia, se empleó la versión 2.1.2 portable para Microsoft Windows, estructurada en cuatro secciones básicas: Menú de herramientas, Árbol de contenidos, Herramientas de edición, Área de trabajo (eXeLearning.net, s.f.). 
Ed. 27. Vol.7 № 4. Diciembre'18 - Marzo'19

DOI: http://dx.doi.org/10.17993/3ctic.2018.62.98-121

En el Gráfico 1 se muestra la distribución de las cuatro secciones de trabajo en las que se encuentra dividido eXe-Learning.

\begin{tabular}{|c|c|c|c|}
\hline Características & \multicolumn{3}{|c|}{ Herramienta tecnológica } \\
\hline Software gratuito & $\begin{array}{c}\text { e earning } \\
\text { Lea }\end{array}$ & $\begin{array}{c}\text { Moodle } \\
\text { M i c r o s o f t } \\
\text { Power Point }\end{array}$ \\
\hline $\begin{array}{c}\text { No requiere conexión a Internet. } \\
\text { Es portable (no requiere instalación) }\end{array}$ & $\mathrm{x}$ & $\mathrm{x}$ & \\
\hline $\begin{array}{c}\text { Diseñado específicamente para contribuir a los } \\
\text { procesos de enseñanza-aprendizaje }\end{array}$ & $\mathrm{x}$ & $\mathrm{x}$ & \\
\hline
\end{tabular}

Tabla 1. Herramientas Tecnológicas -Tabla Comparativa.

Fuente: elaboración propia.

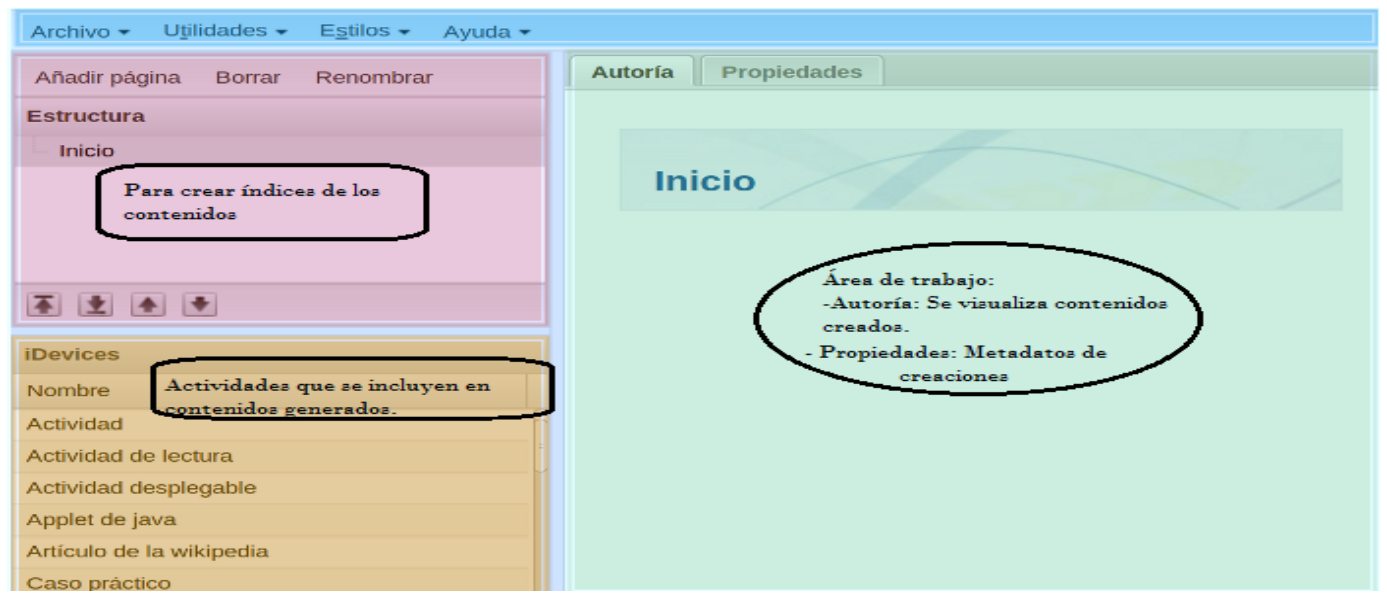

Gráfico 1. Secciones de trabajo del eXe-Learning.

Fuente: eXe-Learning (s.f.)

| 105 
Se aprovechó la condición del eXe-Learning que faculta la creación de una secuencia lógica de páginas que, a manera de un árbol de contenidos, permite planificar didácticamente la estrategia. Los recursos propios de este software educativo (denominados iDevices), facilitaron el desarrollo de las estrategias pre-instruccionales (preguntas dirigidas, actividad focal introductoria, objetivos); co-instruccionales (explorando la Web, estructura textual expositiva, preguntas intercaladas) y post-instruccionales (resúmenes), tomando en cuenta que fue necesario analizar que iDevice se adaptaba mejor al tema a tratar y al tipo de evaluación a aplicar.

Los iDevices fueron seleccionados para desarrollar las estrategias didácticas por su utilidad pedagógica, tal como: información textual, útiles en la presentación de contenidos, imágenes, vídeos; los de actividad no interactiva, aplicados para proponer actividades que incluían retroalimentación; los de actividades interactivas, empleados en la presentación de cuestionarios con retroalimentación y resultados. los de información no textual, utilizados en la presentación de recursos externos (textuales o webs) (eXeLearning 2.1. Tutorial - Manual, s.f.).

Se diseñó la estrategia didáctica de enseñanza, considerando que su estructura contenía los momentos de la clase y en cada uno se detalló la estrategia didáctica a emplearse, el recurso (iDevice) utilizado, el instrumento que empleó el recurso para ejecutarse, la finalidad y las actividades que debían realizar los estudiantes. Finalmente, se incorporó la sección referente a la evaluación, se consideró el tipo de evaluación, los instrumentos de evaluación y los recursos del eXe-Learning utilizados. En la Tabla 2 se presenta la ficha de la estrategia didáctica diseñada para la Clase $\mathrm{N}^{\circ} 1$ de lógica matemática, en esta ficha se perfilan todos los elementos que conforman su estructura y que fueron mencionados anteriormente. La Clase $\mathrm{N}^{\circ} 2$ y a la Clase $\mathrm{N}^{\circ} 3$ se presentan en la Tabla 3 y en la Tabla 4 respectivamente. 
Tabla 2. Estrategia Didáctica - Clase №1.

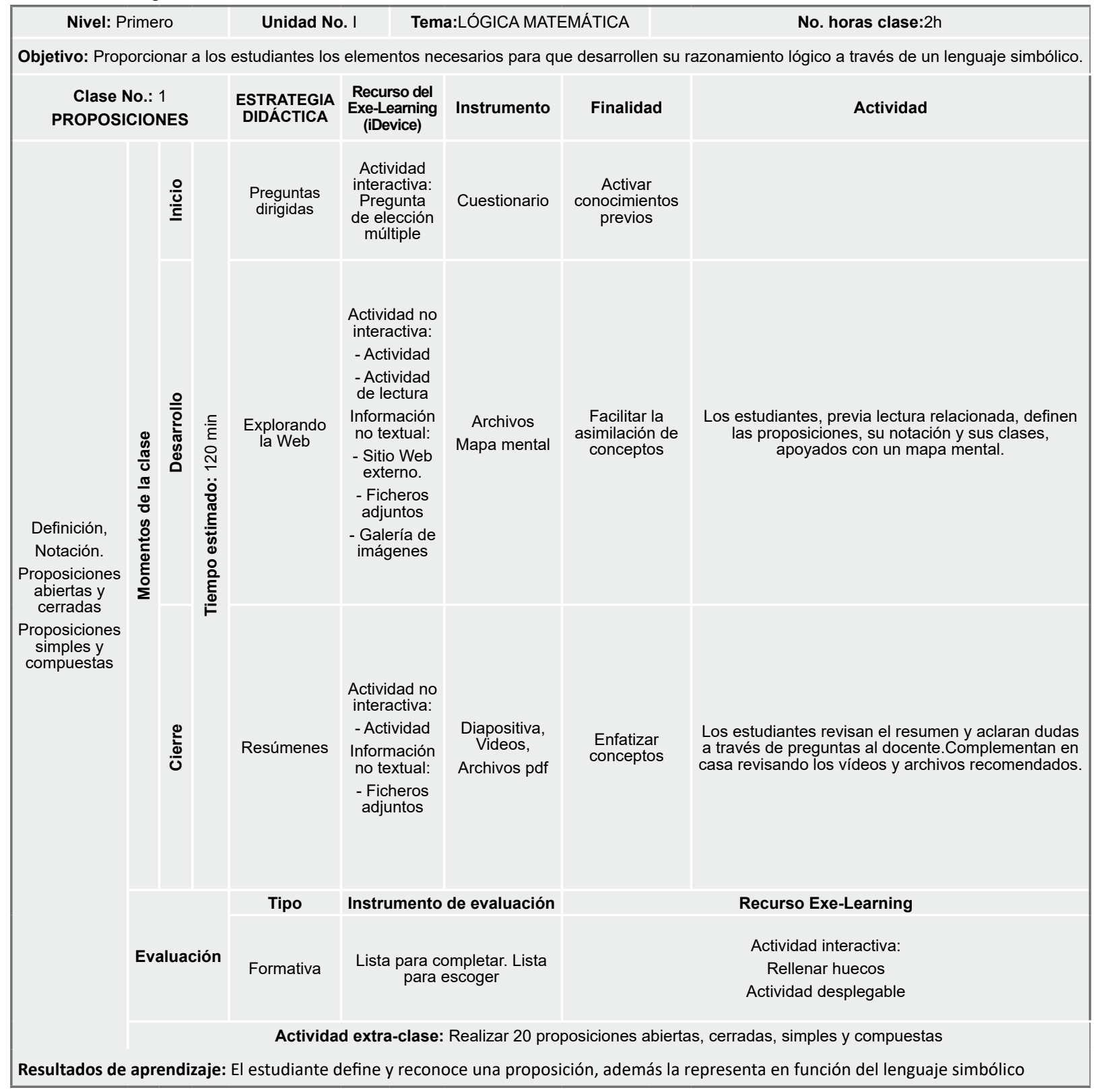


Delineada la estructura de la estrategia se puso en ejecución su creación, se procuró aprovechar la condición de la interfaz gráfica del usuario que dispone el eXe-Learning, presentando en forma esquemática las unidades didácticas seleccionadas para favorecer los procesos cognitivos de los estudiantes; de esta manera, se garantizó la accesibilidad y manejo de este recurso. Incorporadas las unidades, con sus contenidos y actividades, se exportó el proyecto utilizando dos de las varias opciones disponibles; una, que permitió almacenar la información como una página web en una carpeta autocontenida que puede guardarse en el computador o en un dispositivo USB; y la otra, empleando la opción EPUB3 que permitió utilizar el material en un dispositivo móvil. 
Tabla 3. Estrategia Didáctica - Clase $n^{\circ} 2$

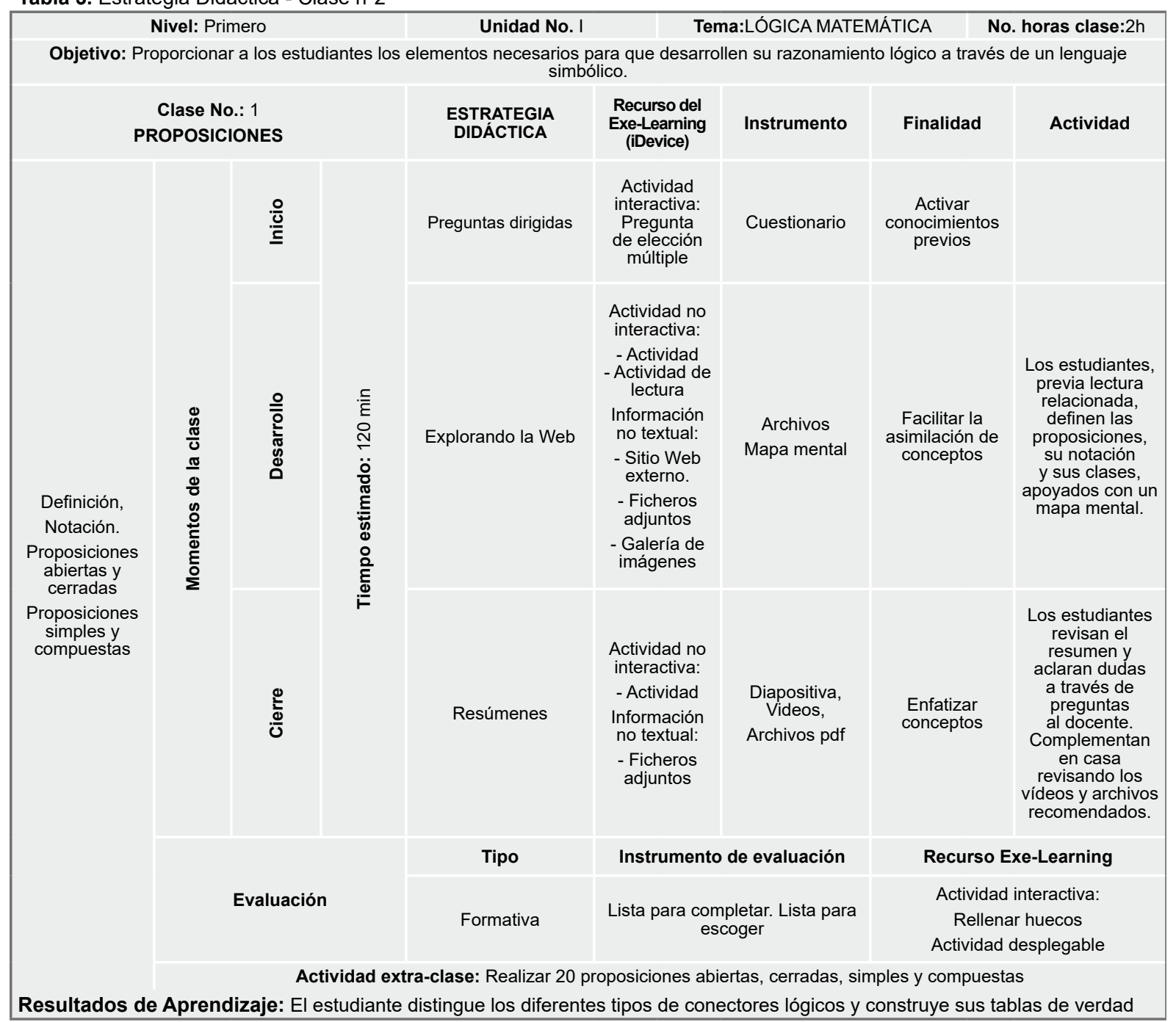

Fuente: Yánez, V. (2016). 
Tabla 4. Estrategia Didáctica - Clase №3.

\begin{tabular}{|c|c|c|c|c|c|c|c|c|}
\hline \multicolumn{4}{|c|}{ Nivel: Primero } & Unidad No. I & Tema: LÓGIC & MATEMÁTICA & \multicolumn{2}{|c|}{ No. horas clase: $2 \mathrm{~h}$} \\
\hline \multicolumn{9}{|c|}{$\begin{array}{c}\text { Objetivo: Proporcionar a los estudiantes los elementos necesarios para que desarrollen su razonamiento lógico a través de un lenguaje } \\
\text { simbólico. }\end{array}$} \\
\hline \multicolumn{4}{|c|}{$\begin{array}{l}\text { Clase No.: } 2 \\
\text { CONECTORES LÓGICOS }\end{array}$} & $\begin{array}{l}\text { ESTRATEGIA } \\
\text { DIDACCTICA }\end{array}$ & $\begin{array}{l}\text { Recurso del } \\
\text { Exe-Learning } \\
\text { (iDevice) }\end{array}$ & Instrumento & Finalidad & Actividad \\
\hline \multirow{6}{*}{$\begin{array}{c}\text { Tipos: } \\
\text {-Negación } \\
\text {-Conjunción } \\
\text {-Disyunción } \\
\text {-Condicional } \\
\text { Bicondicional. } \\
\text { Tablas }\end{array}$} & \multirow{3}{*}{$\begin{array}{l}0 \\
0 \\
\frac{\pi}{0} \\
0 \\
\frac{\pi}{0} \\
0 \\
0 \\
0 \\
0 \\
\frac{0}{0} \\
\frac{1}{0} \\
\frac{0}{2}\end{array}$} & $\stackrel{\frac{O}{0}}{\underline{\underline{O}}}$ & \multirow{3}{*}{ 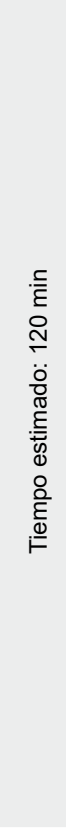 } & $\begin{array}{l}\text { Actividad focal } \\
\text { introductoria }\end{array}$ & $\begin{array}{c}\text { Experimental: } \\
\text { - Ordenar } \\
\text { objetos. } \\
\text { Actividad } \\
\text { interactiva: } \\
\text { - Pregunta } \\
\text { de selección } \\
\text { múltiple. }\end{array}$ & $\begin{array}{l}\text { Plantilla } \\
\text { para ordenar } \\
\text { palabras. } \\
\text { Cuestionario. }\end{array}$ & $\begin{array}{c}\text { Relacionar } \\
\text { información } \\
\text { de la Clase } \\
\text { No.1 con } \\
\text { nueva } \\
\text { información }\end{array}$ & $\begin{array}{c}\text { Los } \\
\text { estudiantes } \\
\text { forman } \\
\text { proposiciones } \\
\text { compuestas } \\
\text { al ordenar } \\
\text { las palabras } \\
\text { y deducen } \\
\text { lo que es } \\
\text { un conector } \\
\text { lógico. }\end{array}$ \\
\hline & & 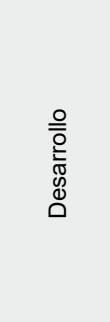 & & $\begin{array}{l}\text { Estructura } \\
\text { textual } \\
\text { expositiva }\end{array}$ & $\begin{array}{l}\text { Actividad no } \\
\text { interactiva: } \\
\text { - Actividad de } \\
\text { lectura }\end{array}$ & $\begin{array}{l}\text { Documento } \\
\text { (archivo } \\
\text { digital). } \\
\text { Cuadro } \\
\text { sinóptico. }\end{array}$ & $\begin{array}{l}\text { Promover } \\
\text { la lectura } \\
\text { comprensiva } \\
\text { y el análisis }\end{array}$ & $\begin{array}{c}\text { Los } \\
\text { estudiantes } \\
\text { organizados } \\
\text { en grupos } \\
\text { realizan } \\
\text { un cuadro } \\
\text { sinóptico } \\
\text { y exponen } \\
\text { sobre la } \\
\text { información } \\
\text { previamente } \\
\text { analizada. }\end{array}$ \\
\hline & & $\frac{0}{20}$ & & Resúmenes & $\begin{array}{l}\text { Actividad no } \\
\text { interactiva: } \\
\text { - Actividad } \\
\text { Información } \\
\text { no textual: } \\
\text { - Ficheros } \\
\text { adjuntos }\end{array}$ & Diapositiva & $\begin{array}{l}\text { Reafirmar } \\
\text { lo expuesto } \\
\text { por los } \\
\text { estudiantes }\end{array}$ & $\begin{array}{c}\text { El docente } \\
\text { presenta un } \\
\text { condensado } \\
\text { de la } \\
\text { información } \\
\text { tratada en las } \\
\text { exposiciones. }\end{array}$ \\
\hline & \multirow{2}{*}{\multicolumn{3}{|c|}{ Evaluación }} & Tipo & \multicolumn{2}{|c|}{ Instrumento de evaluación } & \multicolumn{2}{|c|}{ Recurso Exe-Learning } \\
\hline & & & & Formativa & \multicolumn{2}{|c|}{$\begin{array}{l}\text { Lista de afirmaciones } \\
\text { Cuestionario }\end{array}$} & \multicolumn{2}{|c|}{$\begin{array}{c}\text { Actividad interactiva: } \\
\text { Pregunta verdadero-falso } \\
\text { Rellenar huecos } \\
\text { Actividad desplegable }\end{array}$} \\
\hline & \multicolumn{8}{|c|}{ Actividad extra-clase: } \\
\hline
\end{tabular}

Fuente: Yánez, V. (2016). 


\subsection{DISEÑO DE LA SEGUNDA ETAPA}

Para esta etapa se contó con 26 estudiantes en total, dividiéndolos aleatoriamente en dos grupos, uno de control, que recibían las clases con una estrategia que no empleaba el Exe-Learning; y el grupo experimental, al que se aplicó una estrategia apoyada con el Exe-Learning. Los dos grupos estudiaron los temas: Proposiciones, Conectores lógicos, Lectura y escritura lógicas de la Unidad I - Lógica Matemática, contemplada en el syllabus de Matemática de Primer Nivel, cada grupo recibió 6 horas de clases en total. Luego, los dos grupos fueron evaluados con un sólo cuestionario de 10 preguntas referentes a los temas mencionados y valoradas sobre 1,50 puntos.

Para Gómez (2006), los experimentos 'verdaderos' son aquellos que reúnen los dos requisitos para lograr el control y la validez interna: 1 . Grupos de comparación (manipulación de la variable independiente) y 2. Equivalencia de los grupos. En este caso, la variable independiente (estrategia apoyada con eXe-Learning) fue manipulada al ser aplicada únicamente al grupo experimental y no al grupo control, y la equivalencia de los grupos estuvo garantizada mediante la asignación aleatoria de los estudiantes a cada grupo.

El esquema que representa el diseño de esta investigación se muestra en el Gráfico 2, que indica que tanto el grupo experimental, G1, como el grupo control, G2, han pasado por una asignación aleatoria, R; además, establece que G1 es sometido a manipulación mediante la aplicación de la variable independiente que, en este caso es la estrategia apoyada con el eXe-Learning, y por último, indica que se va a observar los efectos de la manipulación mediante un post-test tanto al grupo experimental como al grupo control, referidos con $\mathrm{O} 1$ y $\mathrm{O} 2$ respectivamente.

Gráfico 2. Esquema del diseño investigación.

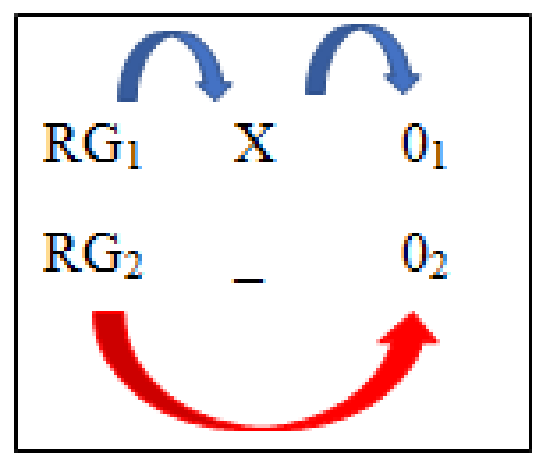

Fuente: elaboración propia. 
Las hipótesis planteadas al inicio de esta investigación fueron las siguientes: Hipótesis nula (H0): No existe una mejora en el desempeño académico de los estudiantes con la utilización del eXe-Learning, como recurso digital de apoyo, cuando se desarrolla una clase de lógica matemática. Hipótesis Alternativa (H1): Existe una mejora en el desempeño académico de los estudiantes con la utilización del eXeLearning, como recurso digital de apoyo, cuando se desarrolla una clase de lógica matemática.

Las hipótesis planteadas fueron contrastadas mediante un análisis estadístico de los resultados obtenidos en las evaluaciones (cuestionario con respuesta de opción múltiple), estableciendo las medidas de tendencia central, y en función de la forma de su distribución y del tamaño de la muestra (que coincide con la población de 26 estudiantes) se eligió la prueba que permitió comparar los datos de las dos muestras y verificar las hipótesis.

\section{RESULTADOS EXPERIMENTALES}

\subsection{RESULTADOS DE LA PRIMERA ETAPA}

Se emplea el recurso del eXe-Learning: actividad interactiva con pregunta de selección múltiple, para determinar los conocimientos previos de los estudiantes. El Gráfico 3a muestra la página de inicio del proyecto, a través de este, el estudiante puede direccionarse al área de interés, preferiblemente siguiendo una secuencia lógica, se puede distinguir el árbol de contenidos de la primera clase. Esta disposición permite un acceso rápido a los temas y actividades planificadas, considerando el momento de la clase (inicio) y la estrategia didáctica, preguntas dirigidas como se muestra en el Gráfico 3b. 


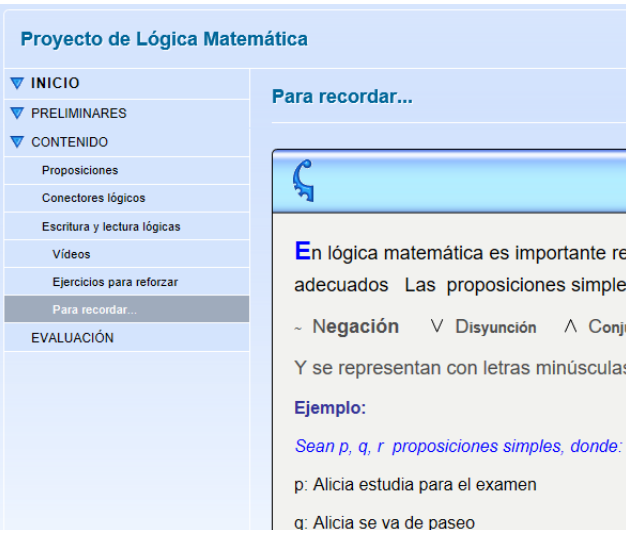

Gráfico 3a. Página de inicio, árbol de contenidos.

Fuente: elaboración propia.

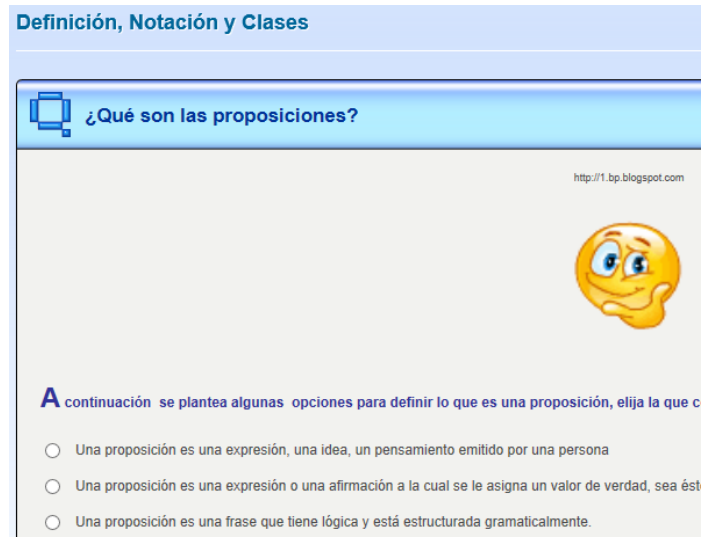

Gráfico 3b. Preguntas de opción múltiple.

Fuente: elaboración propia.

$\mathrm{Al}$ cierre de la clase, está configurada la ventana "Para recordar", que permite visualizar la estrategia de resúmenes y presenta una síntesis de los temas tratados, se muestra en el Gráfico 3a.

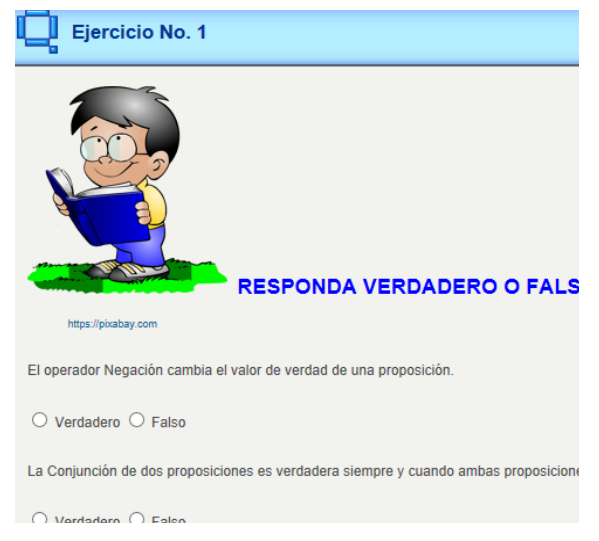

Gráfico 4a. Verdadero y Falso.

Fuente: elaboración propia.

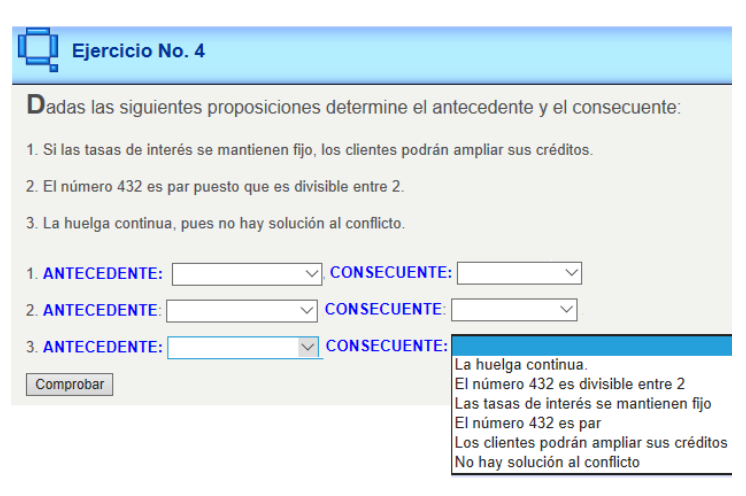

Gráfico 4b. Antecedente y Consecuente.

Fuente: elaboración propia. 
Los gráficos $4 \mathrm{a}$ y $4 \mathrm{~b}$ presentan varias actividades que permitirán realizar la evaluación, tanto formativa como sumativa, se configura con varios recursos del eXe-Learning, como se observa las opciones del recurso son diversas. El Gráfico $4 \mathrm{~b}$ presenta una de las actividades de refuerzo propuestas para consolidar los conceptos planteados, mediante la respectiva retroalimentación, esta sección es parte de la etapa de desarrollo de la clase.

La carpeta autocontenida en la que se encuentra el proyecto puede guardarse en un USB o en un dispositivo móvil. En el caso del dispositivo móvil (teléfono móvil o Tablet) la información se presenta en forma de libro electrónico con índice de contenidos, para visualizar la información se debe tener instalado previamente un lector de este tipo de libros en el dispositivo. El Gráfico 5a permite apreciar que los estudiantes pueden hacer uso de la estrategia a través de varios dispositivos, lo cual demuestra que el eXe-Learning es un recurso digital altamente accesible. Por otro lado, el Gráfico 5b muestra el índice que se genera cuando la carpeta de contenidos del eXe-Learning es guardada en un teléfono celular, esta forma de presentación de los contenidos permite acceder a ellos directamente con sólo hacer clic en la opción requerida.

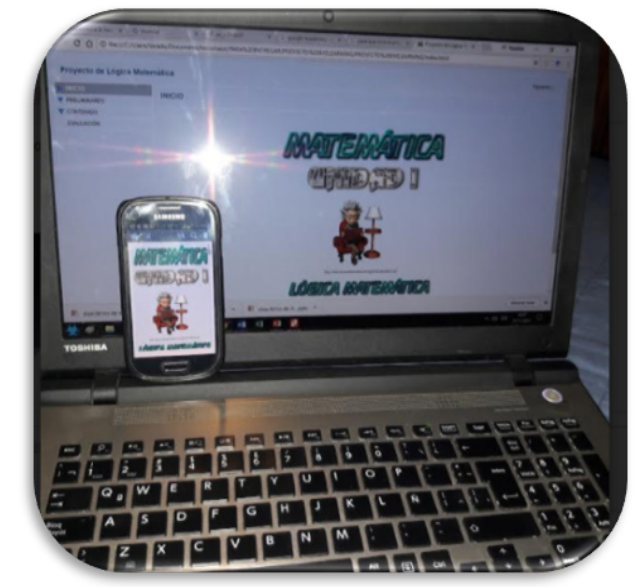

Gráfico 5a. Acceso al proyecto a través de dispositivos móviles.

Fuente: elaboración propia.

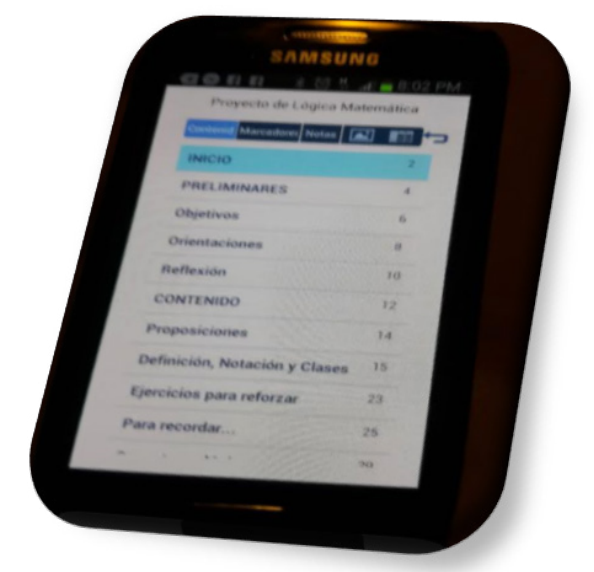

Gráfico 5b. Proyecto presentado como libro electrónico con índice.

Fuente: elaboración propia. 


\subsection{RESULTADOS DE LA SEGUNDA ETAPA}

Los estudiantes tanto del grupo experimental como del grupo control fueron evaluados con un mismo cuestionario de 10 preguntas, los resultados obtenidos se reflejan en la Tabla 5.

Tabla 5. Resultados del cuestionario.

\begin{tabular}{ccc}
\hline No. Estudiante & \multicolumn{2}{c}{ Calificaciones } \\
\hline 1 & Grupo de control & Grupo experimental \\
\hline 2 & 0,60 & 1,50 \\
3 & 0,45 & 0,40 \\
4 & 0,30 & 0,45 \\
5 & 0,45 & 0,85 \\
6 & 1,05 & 1,00 \\
7 & 0,60 & 0,45 \\
8 & 0,45 & 0,85 \\
9 & 0,30 & 0,10 \\
10 & 0,00 & 1,50 \\
11 & 0,00 & 0,50 \\
12 & 1,20 & 1,20 \\
13 & 0,75 & 1,15 \\
& 0,30 & 1,50 \\
\hline
\end{tabular}

Fuente: elaboración propia.

Se analizan los datos de las calificaciones empleando el software estadístico SPSS y se obtuvo los siguientes resultados. La información presentada, establece que la media de 0,8808 y mediana de 0,8500 de calificaciones del grupo experimental (Tabla 6b), son mayores que la media de 0,4962 y mediana de 0,4500 de calificaciones del grupo control (Tabla 6a). Por otro lado, el error estándar de la media del grupo experimental es mayor que aquel del grupo control, lo que también se refleja en los valores de sus respectivas desviaciones estándar; estos resultados indican que las calificaciones del grupo experimental están más alejadas del promedio que las del grupo control. Además, con un error del 5\%, se puede afirmar que las calificaciones del grupo experimental están entre 0.59 y 1.17, y las del grupo control están entre 0.28 y 071 ; teniendo en cuenta que la rúbrica para esta evaluación es de 1.50 puntos, los valores del intervalo del grupo experimental se alejan del óptimo, 
esto se interpreta considerando que si bien los estudiantes tuvieron la oportunidad de utilizar la herramienta eXe-Learning como apoyo para su aprendizaje, si no se preparan adecuadamente para la evaluación, los resultados no pueden ser los mejores.
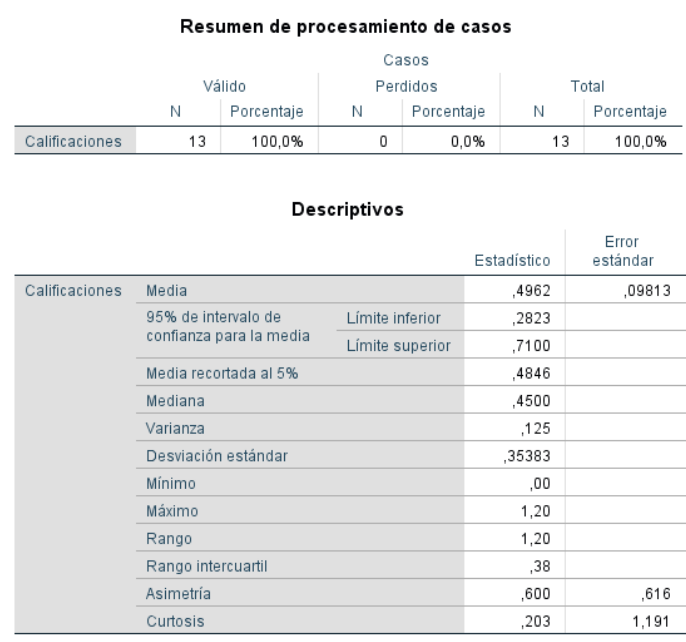

Tabla 6a. Grupo control.

Fuente: elaboración propia.
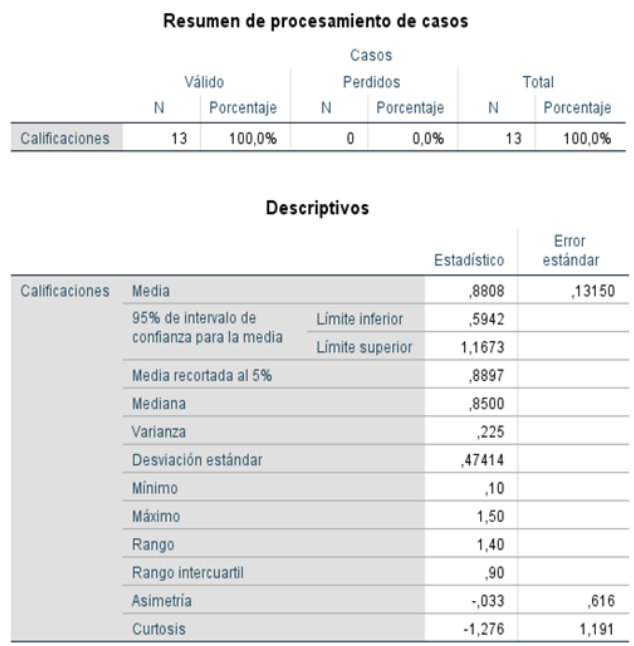

Tabla 6b. Grupo experimental.

Fuente: elaboración propia.

Para probar que la estrategia didáctica apoyada con el eXe-Learning mejora el desempeño académico de los estudiantes es una afirmación válida, se recurre al análisis estadístico; primero para determinar si los datos tienen una distribución normal, y dependiendo de ello, para determinar la prueba estadística a aplicarse (paramétrica o no paramétrica). Con este fin se establece una variable aleatoria (variable numérica = calificaciones de la prueba); y una variable fija (que crea los dos grupos); además, se considera muestras independientes sometidas a un estudio transversal (se analizan en un mismo momento). Bajo estas condiciones, se plantea demostrar que la variable aleatoria en ambos grupos se distribuye normalmente; para lo cual se emplea la prueba de Shapiro-Wilk, considerando que el tamaño de las muestras es menor a 30 y asumiendo un nivel de significancia () igual a 0,05. El planteamiento en función de la hipótesis es el siguiente:

- Ho $=$ Los datos provienen de una distribución normal.

- H1 = Los datos No provienen de una distribución normal. 
Se acepta Ho si el valor de probabilidad es $\geq$; y se acepta H1 si el valor de probabilidad es <.

Tabla 7. Análisis de la distribución normal.

\section{Prueba de normalidad}

\begin{tabular}{llr|r|r} 
& \multicolumn{2}{c}{ Grupos de estudiantes } & & \multicolumn{3}{c}{ Shapiro-Wilk } \\
\cline { 1 - 4 } Calificaciones & Grupo de control & & \multicolumn{1}{c}{ gl } & \multicolumn{1}{c}{ Sig. } \\
\cline { 2 - 5 } & Grupo experimental &, 935 & 13 &, 391 \\
\hline
\end{tabular}

Fuente: elaboración propia.

Como se muestra en el Tabla 7, el valor de probabilidad emitido por la prueba de Shapiro-Wilk, tanto para el grupo control como para el experimental es igual a 0,391 y a 0,264 respectivamente, y en cada caso resulta ser mayor que $\alpha=0,05$; por lo tanto, se acepta Ho, es decir, que los datos provienen de una distribución normal. Con este resultado y las característica de las muestra, se determina que la prueba idónea para el manejo estadístico de los datos es la prueba paramétrica de $t$ de Student. No se podía determinar el nivel de significancia en la Prueba de t de Student, sin antes corroborar el estado de las varianzas, a través de la Prueba de Levene, para lo cual se planteó lo siguiente:

- Ho = Las varianzas son iguales.

- H1 = Existe diferencia significativa entre las varianzas.

Si $\alpha=0.05$, se acepta Ho si el valor de probabilidad es $\geq$; y se acepta H1 si el valor de probabilidad es $<\alpha$.

Los resultados de la Prueba de Levene, establecen que el nivel de significancia es igual a 0.161 y es mayor a $\alpha=0.05$; por lo tanto, se acepta Ho, es decir, se confirma que las varianzas son iguales. Con esta información, y tomando en cuenta las hipótesis consideradas al inicio de esta investigación, se replantea, y se propone la siguiente hipótesis que está relacionada con los resultados estadísticos específicos mencionados:

"La media de las calificaciones de la prueba aplicado a los estudiantes del grupo experimental es mayor que la media de las calificaciones aplicada a los estudiantes del grupo de control”. 
H1 = Existe una diferencia significativa entre la media de calificaciones del grupo experimental y la del grupo de control.

Ho $=$ No existe una diferencia significativa entre la media de calificaciones del grupo experimental y la del grupo de control.

Criterio: $\mathrm{Si} \alpha=0.05$, se acepta Ho si el valor de probabilidad es $\geq$; y se acepta $\mathrm{H} 1$ si el valor de probabilidad es $<\alpha$.

Tabla 8. Análisis con la prueba paramétrica t de Student para muestras independientes.

\begin{tabular}{|c|c|c|c|c|c|c|c|c|}
\hline & & & & & bat para la igual & lad de medias & & \\
\hline & & & & & & Diferencia de & $\begin{array}{l}\text { 95\% de interva } \\
\text { de la di }\end{array}$ & $\begin{array}{l}\text { le confianza } \\
\text { ncia }\end{array}$ \\
\hline & & $t$ & او & Sig. (bilateral) & $\begin{array}{l}\text { Oiterencla de } \\
\text { medias }\end{array}$ & $\begin{array}{c}\text { errtor } \\
\text { estándar }\end{array}$ & Inferior & Superior \\
\hline Calificaciones & $\begin{array}{l}\text { Se asumen varianzas } \\
\text { iguales }\end{array}$ & $-2,344$ & 24 & .028 & -38462 & ,16408 & -72327 & -04597 \\
\hline & $\begin{array}{l}\text { No se asumen varianzas } \\
\text { iguales }\end{array}$ & $-2,344$ & 22,202 & .028 & -38462 & 16408 & -,72472 &,- 04451 \\
\hline
\end{tabular}

Fuente: elaboración propia.

El valor de probabilidad emitido por la prueba de t de Student, mostrado en la Tabla 8, asumiendo la igualdad de las varianzas, es 0,028 y es menor que $\alpha=0,05$; por lo tanto, se aceptó H1 que dice: Existe una diferencia significativa entre la media de calificaciones del grupo experimental y la media de calificaciones del grupo de control.

\subsection{INTERPRETACIÓN}

La media de las calificaciones de la prueba aplicado a los estudiantes del grupo experimental es mayor que la media de las calificaciones de la prueba aplicado a los estudiantes del grupo de control; es decir, esta diferencia no es producto del azar y da a entender que una estrategia didáctica de enseñanza, apoyada con una herramienta tecnológica como el Exe-Learning, ayuda a mejorar el desempeño académico de los estudiantes de Matemática de Primer Nivel. 


\section{CONCLUSIONES}

El eXe-Learning como recurso digital de una estrategia de enseñanza-aprendizaje de una clase de Matemática, presenta una gama de posibilidades (iDevices) con las que se puede contar para diseñar la estrategia. Así, para la presentación de contenidos, imágenes, vídeos es útil el iDevice Información Textual; para diseñar actividades que incluyen retroalimentación se puede emplear el iDevice Actividad No Interactiva; si se quieren presentar cuestionarios con retroalimentación y resultados, se puede emplear el iDevice Actividades Interactivas; y para la presentación de recursos externos se puede utilizar el iDevice Información No Textual. Sin embargo, es el docente quien con un criterio apropiado y en función de los objetivos que desee alcanzar, adaptará las opciones disponibles a los planteamientos de su estrategia.

La versatilidad de los iDevices permiten diseñar estrategias como: preguntas dirigidas, actividad focal introductoria, objetivos (pre-instruccionales); explorando la Web, estructura textual expositiva, preguntas intercaladas (co-instruccionales) y resúmenes (post-instruccionales); teniendo en cuenta que es necesario analizar que iDevice se adapta mejor al tema a tratar y al tipo de evaluación a emplear.

$\mathrm{Al}$ aplicar la estrategia didáctica para la enseñanza de matemáticas apoyada con el eXe-Learning a los estudiantes de primer nivel de Ingeniería de Sistemas de la Pontificia Universidad Católica del Ecuador Sede Esmeraldas, se obtuvieron resultados que se reflejaron en las calificaciones de una evaluación (con una rúbrica de 1.5 puntos), en la que los estudiantes que recibieron las clases apoyadas con el eXe-Learning (grupo experimental) estuvieron entre 0.59 y 1.17 puntos; en cambio que los estudiantes que recibieron sus clases sin este recurso tecnológico (grupo control), presentaron calificaciones entre 0.28 y 0.71 puntos. Conforme al análisis realizado con técnicas estadísticas inferenciales paramétricas desarrolladas con el software SPSS, se determinó que estos valores no son producto del azar y más bien confirman que el empleo del eXe-Learning, contribuye a mejorar el desempeño académico de los estudiantes.

Los estudiantes son nativos digitales, por lo tanto, tienen facilidad para manejar la herramienta educativa. No obstante, su manejo no es suficiente para mejorar su rendimiento académico, ellos deben estar comprometidos a construir su conocimiento apoyándose en el eXe-Learning, es decir deben estudiar responsablemente los temas y realizar las actividades propuestas, en definitiva, deben estar predispuestos a aprender. Lo mencionado, se evidencia al observar el límite superior 
(1.17 puntos) de calificaciones obtenidas por los estudiantes del grupo experimental que se aleja del valor óptimo (1.50 puntos).

Por los resultados obtenidos, y dado que la población considerada fue de 26 estudiantes, sería importante ampliar el estudio, considerando otros niveles de la misma carrera o el mismo nivel en diferentes carreras de la PUCE Esmeraldas, esto ayudaría a validar los resultados y garantizar su generalización. Obviamente se requiere crear una estrategia diferente en cada caso, lo que tendrán en común es el uso del eXe-Learning como recurso digital.

\section{REFERENCIAS}

Campos Campos, Y. (2000). Estrategias didocticas apoyadas en tecnologra. México D.F., México: DGENAMDF.

Carrasco, J. (2004). Estrategias didácticas personalizadas (1): Conceptos y tipos. En:J. Carrasco, Una didбctica para hoy: cymo ensecar mejor (p. 83). Madrid, España: RIALP.

Castillo, S. (2008). Propuesta pedagógica basada en el constructivismo para el uso óptimo de las TIC en la enseñanza y el aprendizaje de la matemática. Revista latinoamericana de investigaciyn en matemб́tica educativa, 11(2), pp. 171-194. Recuperado de: http://www.scielo.org.mx/scielo.php?script=sci_ arttext\&pid=S1665-24362008000200002

Castro, E., Peley, R., y Morillo, R. (2006). La práctica pedagógica y el desarrollo de estrategias instruccionales desde el enfoque constructivista. Revista de Ciencias Sociales, 12(3), pp.591-595. Recuperado de: http://www.scielo.org.ve/scielo.php?script=sci_arttext\&pid=S1315-95182006000300012\&lng=es\&tl $\mathrm{ng}=\mathrm{pt}$.

Díaz Barriga, F. y Hernández Rojas, G. (2010). Estrategias docentes para un aprendizaje significativo. Una interpretaciyn constructivista (3 ed.). México, D.F., México: Mc Graw Hill.

eXeLearning.net. [en línea]. (s.f.). [Consulta: 1 de noviembre de 2017]. Recuperado de: http:// exelearning.net/caracteristicas/\#tab1 
eXe-Learning 2.1. Tutorial - Manual. [en línea]. (s.f.). [Consulta: 6 de diciembre de 2017]. Recuperado de: http://exelearning.net/html_manual/exe20/idevices_de_informacin_no_textual.html

Ferro Soto, C., Martínez Senra, A., y Otero Neira, M. C. (2009). Ventajas del uso de las TICs en el proceso de enseñanza-aprendizaje desde la óptica de los docentes universitarios españoles. Edutec. Revista Electrynica De Tecnologra Educativa, (29), a119. Recuperado de: http://www.edutec.es/revista/ index.php/edutec-e/article/view/451

García, A., Escalera, M., y Navarro, R. E. (2011). Variables asociadas con el uso de las TIC como estrategia didáctica en el proceso enseñanza-aprendizaje de la Matemática Financiera. Una Experiencia desde el aula de clase mejora. Revista Iberoamericana de Evaluaciyn Educativa, 4(2), pp. 118135. Recuperado de: https://dialnet.unirioja.es/servlet/articulo?codigo=4511204

Gómez, M. (2006). Introducciyn a la metodologra de la investigaciyn cientufica. Córdoba, Argentina: Brujas.

González, A. (2016). Los jóvenes, las TIC, y primer año en la universidad. Trayectorias Universitarias, 2(3), pp. 1-8. Recuperado de: https://revistas.unlp.edu.ar/TrayectoriasUniversitarias/article/view/3023/2773

Mora, C. (2003). Estrategias para el aprendizaje y la enseñanza de las matemáticas. Revista de Pedagogra, 24(70), pp. 181-272. Recuperado de: http://www.scielo.org.ve/scielo.php?script=sci_ arttext\&pid=S0798-97922003000200002\&lng=es\&tlng=es.

Oyola, M. y Gómez, I. (2012). Estrategias didácticas basadas en el uso de TIC aplicadas en la asignatura de física en educación media. Escenarios, 10(1), pp. 17-28. Recuperado de: http://dialnet. unirioja.es/servlet/articulo?codigo $=4495590$

Sánchez, S. D. C. N., Perera, J. J. D., y Pérez, H. H. (2016). Estrategias de enseñanza para las matemáticas en el nivel superior. Revista Iberoamericana de Producciyn Acadümica y Gestiyn Educativa, 3(5). Recuperado de: http://www.pag.org.mx/index.php/PAG/article/view/434

Yánez Ortiz, V. (2016). TIC's como estrategia didбctica en el aprendizaje de la Matemótica de primer nivel de ingenierma en gestiyn ambiental. (Trabajo de fin de Máster). Pontificia Universidad Católica del Ecuador Sede Esmeraldas, Ecuador. 